\title{
CONVERSION OF VIRTUAL REALITY INTO A MIXED REALITY
}

\author{
Ivan Pinćjer (D), Neda Milić (D), Irma Puškarević (iD), Nada Miketić (iD) \\ University of Novi Sad, Faculty of Technical Sciences, \\ Department of Graphic Engineering and Design, Novi Sad, Serbia
}

\begin{abstract}
Nowadays technology has developed a new form of appearance - virtual reality. This term currently exists in three different forms, virtual reality, augmented reality and mixed reality. Among these technologies, mixed reality has the most potential for use in everyday activities and education. This paper represents the overview of virtual reality technologies, their functioning and potential applications. When it comes to interaction, the mixed reality devices provide a completely new way of perception and understanding of the virtual space and objects. Since it is possible to interact with objects that exist in a virtual world in the same way as with objects that exist in the physical world, the high potential for the use of this technology for learning is evident. Utilizing advanced hardware components such as transparent displays and cameras simulating stereoscopic vision, in combination with advanced software technologies including spacial mapping, ambient lighting, ambient sound, identifying objects, movements from the real world and location, MR provides the closest $\mathrm{HCl}$ experience so far. Discussing the potential use of this technology, and current development phase in which it exists, the paper has the aim to induce questions and interest about further development of its use and application, such as multimedia learning (Mayer. 2009). The example of the use of MR technology on the HoloLens device is provided in this paper, in order to demonstrate the way of its use.
\end{abstract}

Key words: virtual reality, mixed reality, holograms, 3D modelling

\section{INTRODUCTION}

The moment we find ourselves irresistibly reminds us of the time when the first computers were created. Today we use them in ways that could not even be imagined. They were used exclusively for scientific purposes and to speed up the calculation. Their size was occupying the entire floors of the universities in which they were located. The borders that computers will reach are still not determined. The same situation can be recognized today in a "new" idea. Holograms are something that is part of science fiction, but also of everyday life. As a security measure, on the bills they became irreplaceable. However, the desire to record and reproduce the holographic animated image is still an unachievable goal. Nevertheless, the benefits of the holographic display can still be achieved with the help of high-end computing technology and the latest stereoscopic display that allows a virtual 3D projection.

As in the mid-20 ${ }^{\text {th }}$ century, computers were large and not easy to use, nowadays, holographic devices can be found that began their global conquest in the same way. Devices that allow the illusion of a holographic display is now made in the form of spectacles. Spectacles are, de facto, a whole computer with a plethora of different cameras and sensors, which in real-time maps the space around the users and put virtual models in it. As well as the first computers, these devices are waiting for improvement, fuelled by the versatility of the application.

Besides Microsoft, the world's largest corporations such as Apple, Google, Facebook, Disney, Amazon and many others are greatly developing their applications, to take advantage of this development of computer technology. It will not take much time until the virtual objects inserted into the real world become an everyday asset as e-mail or smartphones.

Since the digital world has become necessary, there is a great need to find ways to better interact with it. External devices such as mice and devices with touchscreen technology limit the interaction with the digital world to the 2D plane. Human-computer interaction is transmitted to a $3 \mathrm{~d}$ real world, through virtual holograms. Of course, in the real world, we can use our own body to manipulate digital content.

In this mixed reality, the virtual space is replaced by the real world in which the user is aware of his real environment (not lost in space), which is a more natural environment for day-to-day tasks, such as work, learning or interacting with other people.

Mixed reality will enable digital content management in the same way as we manipulate real-world objects in a real environment. Finding the simplest solution for this kind of interaction with the digital world will show the path for further development of computer sciences. 
As the big breakthroughs were made in the field of virtual reality (Tavanti et al, 2001), it would be a big step backwards if 3D models from VR could not be customized and used in an augmented or mixed reality. The way this transition can be done will be shown in this paper.

\section{THE IMPORTANCE OF INTERACTION IN EDUCATION}

Interactivity is the term emerged alongside new technologies and development of personal computers, and it is present in everyday life activities. Omnipresence of technologies in the everyday environment, including homes and schools, and its development aid people activities and different jobs. This led to the development of different habits and an increased necessity for interactivity with objects. Furthermore, interactivity is an essential characteristic, especially when it is utilized with learning new skills and acquiring new knowledge. Researchers who have studied learning problematic have identified interactivity as a key contributing factor to better learning. One of the first proposed theories by Bruner (1966), claims that learning is an active process and that learners construct their understanding of the subject when involved in the activity and build their knowledge based on existing knowledge and prior experience. According to Phil Race (2014), knowledge is just information for the student. The information becomes knowledge when something is done with it. This means that student has to apply, calculate, compare, analyse, interpret, integrate information in order to create individual knowledge. Creation of knowledge is an active process which educator facilitate through finding possibilities for learning. This opens an opportunity for combination of traditional approaches to education and interactive distance learning.

Additionally, it is important to emphasize that feedback is a key element of interaction (Oxland, 2004), whereas according to the constructivist theory of active learning interaction is a basic prerequisite for effective learning. Digital era and electronic devices ensured conditions for utilization these theories in their full potential. The form in which these theories found its use, is a new field of research called Human-Computer Interaction $(\mathrm{HCl})$. $\mathrm{HCl}$ is an inevitable part of many scientific disciplines such as computer sciences, graphic design, architectural planning, mechanical engineering et cetera. $\mathrm{HCl}$ can be considered as the tool designed to mediate between interaction human being and their environment. Concepts included in the $\mathrm{HCl}$ field of research are a presentation of information to the user, mental models, the possibility for the user to control the system, input devices, and user interface. Technology as an aid for performing various activities can be considered as a tool. The idea behind the tool as a mediator is that they along with humans form "functional organs". Therefore, the combination of natural human abilities and capacities of external components - tools - provide conditions for performing a task or function much more efficiently. The analogy of this is that human eye aided with glasses form functional organ that provides better sight. Nowadays, there is a constant tendency for $\mathrm{HCl}$ to improve, to become as closest as possible to humans, immerse entirely in their lives and become insensible. Technologies that enable this are: $V R, A R$ and, $M R$, which will be discussed follows.

\section{NEW TECHNOLOGIES AND TYPE OF INTERACTION WHICH THEY PROVIDE $(V R, A R, M R)$}

Technologies in which $\mathrm{HCl}$ is highlighted and provides an experience that erases barrier between virtual and physical world are:

- Virtual reality (VR)

- Augmented reality (AR)

- $\quad$ Mixed reality (MR)

Virtual reality - applies to a group of technologies that make the connection between the real and virtual world in "virtual continuum" that blends real environment with virtual. The main characteristic of VR technologies is that the observer is entirely immersed in a virtual world without the opportunity to see the real-world environment. The observer can have interaction with the virtual world in an approximately identical way as with the real world. Virtual reality emulates properties of the real world laws of physics, time, space, mechanics, material properties - which is at the same time the main advantage of this technology. It was widely spread as a tool for educational purposes (Barraclough et al, 1998), (Pinćjer et al, 2018). 
Augmented reality - these technologies also connect virtual and real content. However, they retain the possibility of observing the real world environment and sense of it. AR technology enables that world becomes improved, refined with digital computer generated content, most commonly with a virtual object which covers real-world environment in the sense of additional digital layer. This technology enables interaction with a user as well.

Mixed reality - also integrates physical and virtual reality retaining the perception of the real-world environment. The main characteristic of $M R$ is the representation of the virtual environment and objects in it conjoint on one display. MR technology exists between two ends of the virtual continuum - realworld environment and virtual reality.

\section{MIXED REALITY}

As it is previously explained, MR is created through the integration of real physical world and digital world, in the way that digital objects are entirely adapted and perceived as if they are the part of the physical world. MR bridges the gap between physical reality and virtual reality and makes that these two are perceived simultaneously as one world. The three components are required for MR experience to exist: human, computer and environment.

Digital objects are imported in the real world as a hologram using stereoscopic projection and addition of a light in front of the observer's eye. The more accurate term for holograms could be virtual holograms because they are not visible in the real world environment without special equipment - glasses. One of these special glasses for mixed reality perception is Microsoft HoloLens glasses.

HoloLens projects holograms onto transparent displays within glasses. With environment mapping, holograms are bound with real objects and positioned in the real world that is visible through display glasses. Each eye individually receives a stereoscopic image, adding the illusion of three-dimensionality to holograms. Virtual holograms are objects defined with light and sound. They can react on users look, body gestures and voice commands. Holograms can even have interaction with real-world objects and surfaces which encircles user, enabling them to transfer digital objects in the real world.

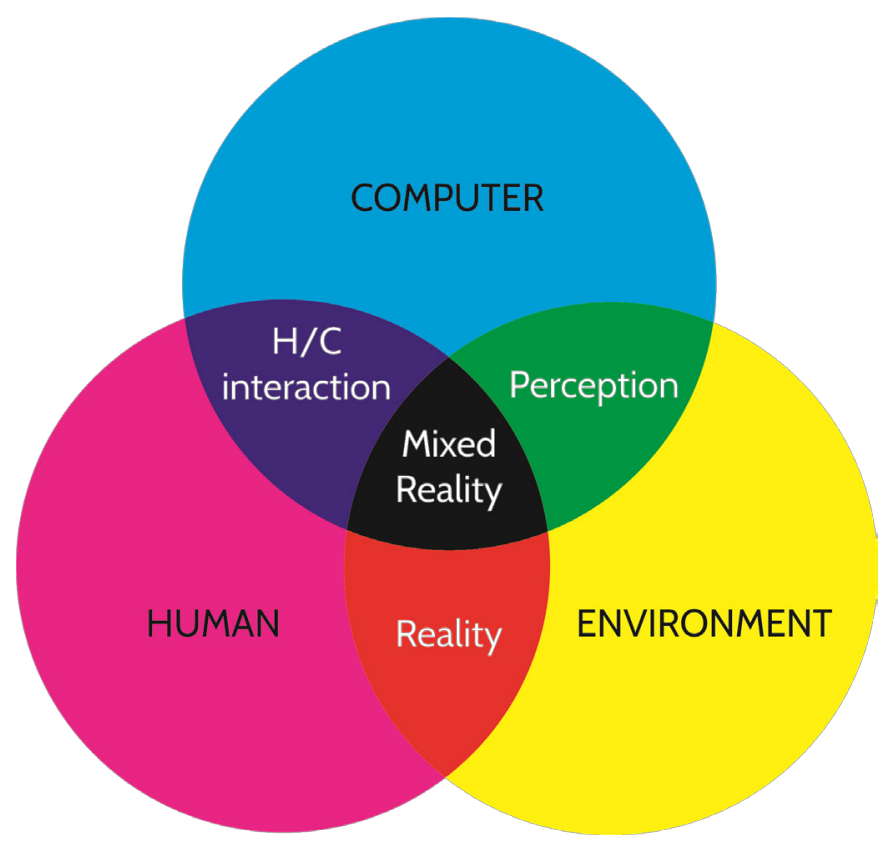

Figure 1: Mixed Reality binding Human, Environment and Computer (Bray, Zeller. 2018)

The realism of the hologram depends on its application. Games require holograms that will look like cartoon models, while simulations involve digital objects high level of realism. To make the digital world more realistic in real life, the Microsoft HoloLens use sound. Speakers are placed above the user's ears, allowing them to hear virtual digital objects that bounce off the real world surface or even hear objects behind them. This creates an illusion that virtual objects are really around the user. In this way, the 
feeling of depth of space increases. As the speakers do not overlap the user's ears, they only add sound to the surrounding sound without blocking it.

Mixed reality devices with transparent displays show holograms by adding light to the real world. As the displays work on the additive system of colour mixing, where the black is obtained by the absence of light, it is clear that in the case of transparent displays, the absence of light will show the background, i.e. the environment. For this reason, the black pixels on the model will be completely transparent; they will not be displayed. This is an essential feature that must be taken into account when creating 3D models. Depending on the background even white pixels may be somewhat transparent.

In order to achieve the effect of depth, several techniques can be used. First one is to position the hologram to the places you would expect to find it in the real world. The realism of the mug hologram is much greater if the hologram is positioned on the table, where it would be expected to see mug, instead of floating somewhere in the air. In addition to mapping the surface on which a virtual hologram could stand, realism can also allow the setting of a shadow that would indicate that the object is in the real world. Adding a shadow is not as easy task, because if we remember, holograms work on the principle of adding a light to the world, and they cannot take it away to create a shadow. In order to achieve the shadow effect, it is necessary to do so call a negative shadow. It is achieved by first placing the mild halo effect around the virtual object in the form of the illuminated surface around the object. This illuminated surface is also a hologram and turning off the lighting in the appropriate places the shadow impression is obtained.

The holograms can be positioned, by the user, anywhere in the room. It is recommended that they are placed at approximately 2 meters from the users. The impact of the hologram's reality will be reduced as it gets closer to the user. Optimal area for placing the holograms is from 1 to $5 \mathrm{~m}$.

The impression of reality is further achieved by the ability of holograms to go behind real world objects. For example, a holographic robot can find the door in the room and simply go through them, disappearing in the hallway. For the holographic devices to be successful in this type of tasks, they must be aware of their environment. It is this awareness that has enabled the use of virtual holograms in real space.

Several technologies are involved in the creation of MR. The MR device can detect environmental information using perception APIs (Application programming interface). The device can detect and receive information from an environment, such as a person's position in the area by monitoring head movements, spatial mapping, ambient lighting, ambient sound, identifying objects and location. A very important aspect of the MR technology application is the ability to translate movement from the physical world into the virtual in real time.

Mixed reality devices can distinguish different surfaces in the real world such as floor, walls, tables or chairs. This feature helps them set up virtual models at appropriate locations, creating constraints. Thus, virtual holograms can use these surfaces to move around or even sit on them.

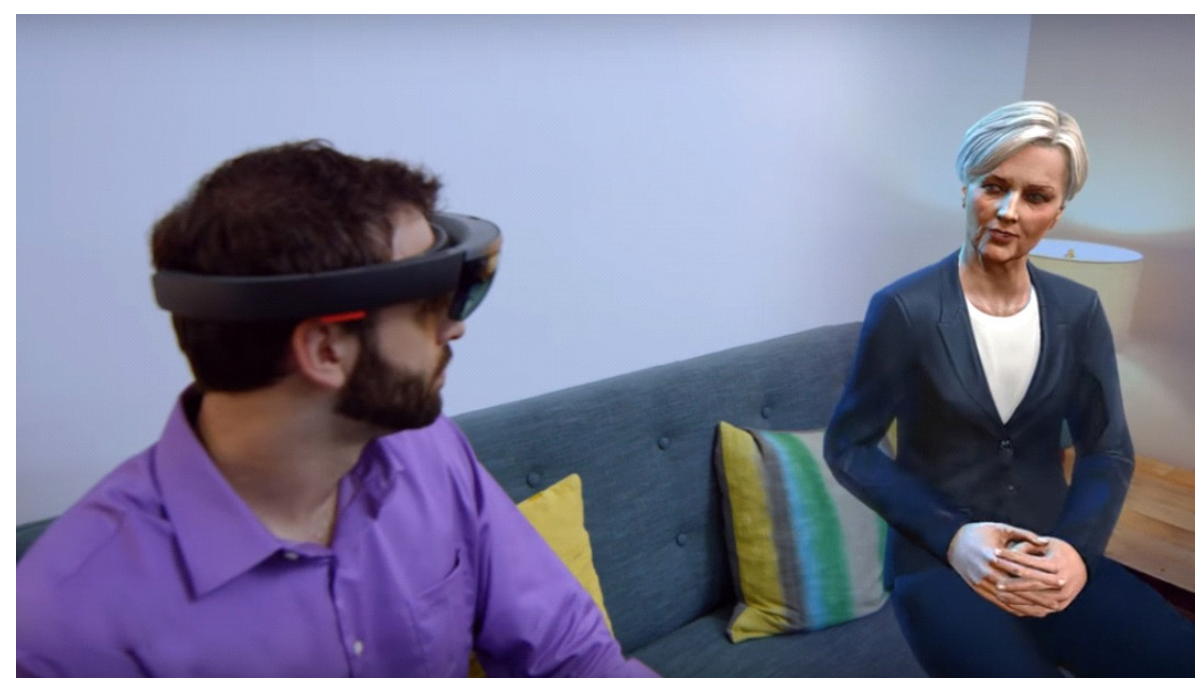

Figure 2: Holograms using a real-world object, furniture (Printscreen: https://www.youtube.com/watch?time_continue=17 HYPERLINK L 
Mapping of the environment is one of the first steps in the process of preparing the application for particular tasks. During the mapping, the user indicates the area in which the hologram will appear. Mapping begins by a user looking at the environment and passing through it. In this process, the topology of an area is created. The more time is one spent in looking to the area; the area will be mapped with more details. Depending on the application's requirements, when the desired coverage is reached, the holograms can be placed in the mapped area. Mapping data can be saved and used on other occasions or even transferred to another device.

Based on data received from the sensor, the software determines the surface characteristics. The pathway taken by the camera during the scanning process is also taken into account, and the software can assume that the surface is a floor, etc. As devices for mixed reality are in the development phase, raw data can be taken from the sensors and used in the analysis and preparation of new applications.

\section{CREATION OF VIRTUAL HOLOGRAM}

Nowadays, technology (hardware and software) is available more than ever. The "ordinary" user is given the opportunity to deal with areas that, until recently, were considered unachievable for broad masses. This is also the case with software for creating a virtual hologram for mixed reality. However, in this technology, the hardware is still not present in people's homes, as is the case with personal computers. Independently of the hardware, with the appropriate software, it is possible to create a hologram that can be transferred later and used on the desired device.

\section{REQUIRED STEPS FOR VIRTUAL HOLOGRAM CREATION}

\subsection{D model}

For enabling holograms to be seen in space, it is necessary to make them in digital form, as a 3D model. Regardless the model is an asset from the internet or created by the author it can be in the same way adapted for the use on the HoloLens device.

\subsection{Unity software}

Unity engine for creating video games, applications and interactive content generally, is necessary to use to adapt the model for the display on the HoloLens device. Unity also enables making the build of the app that needs to be deployed in the HoloLens device.

\subsection{Visual Studio}

For the creation of the app, for debugging and deployment on the device Visual Studio framework is required. In the Unity framework, it is possible to deploy the app on various devices such as computers, mobile phones, or any remote devices including HoloLens glasses. Also, the Visual studio provides a big advantage because it is possible to test and simulate the appearance and behaviour of the app on the chosen device. 


\section{TRANSFERRING 3D MODELS TO A VIRTUAL HOLOGRAM ON MICROSOFT HOLOLENS}

An example illustrates the conversion of a virtual 3d model of a printing press Risograph - Riso EZ 570 to the virtual hologram on the Microsoft HoloLens MR device. A 3D model can be created in any 3D modelling software; an example model given here is created in the $3 \mathrm{ds}$ Max.

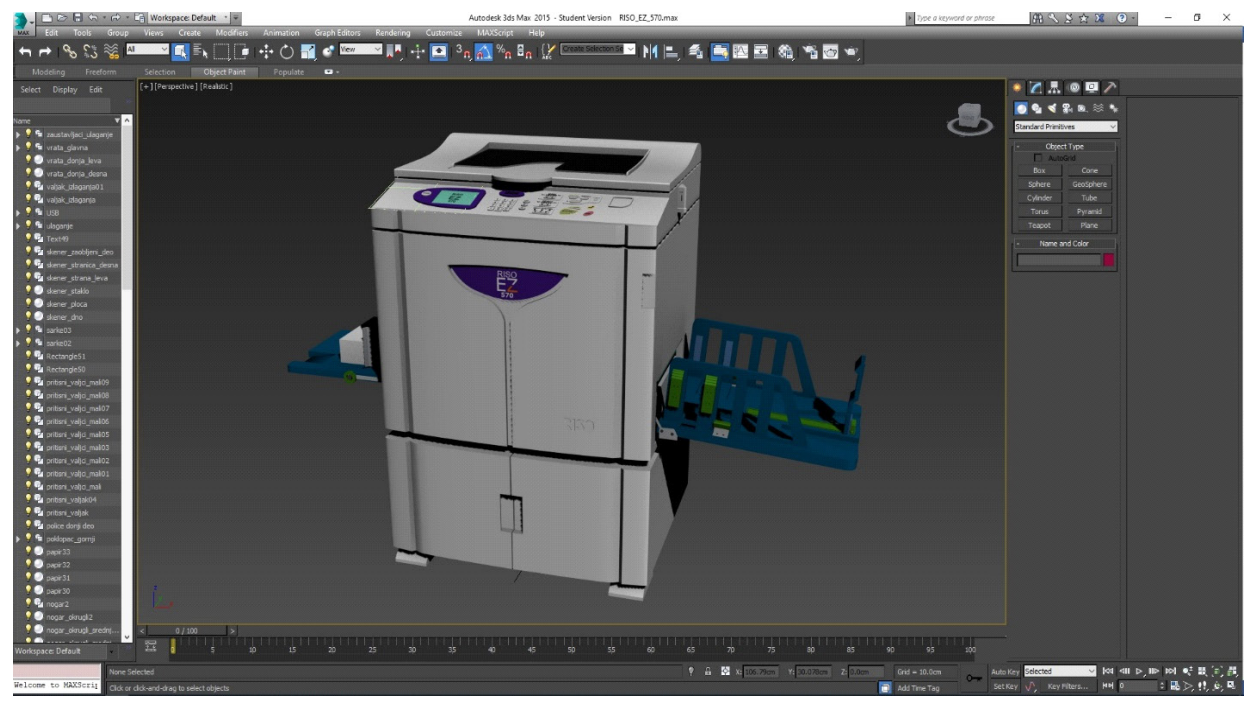

Figure 3: The virtual digital machine in 3ds Max

To import the model into Unity software, it is necessary to export it appropriately. This can be done in many ways, but the most convenient way is to export to the FBX exchange format. The reason for this is that this format keeps the full accuracy of the display and functionality of the object. This format stores information about all the parameters and elements that are present in the 3D scene: lights, textures, animations, object deformation, the position of normals, and more.

Therefore, the example object is saved as an FBX file.

Then it is necessary to make certain settings in Unity software so that the object can be adjusted to the display on the HoloLens device.

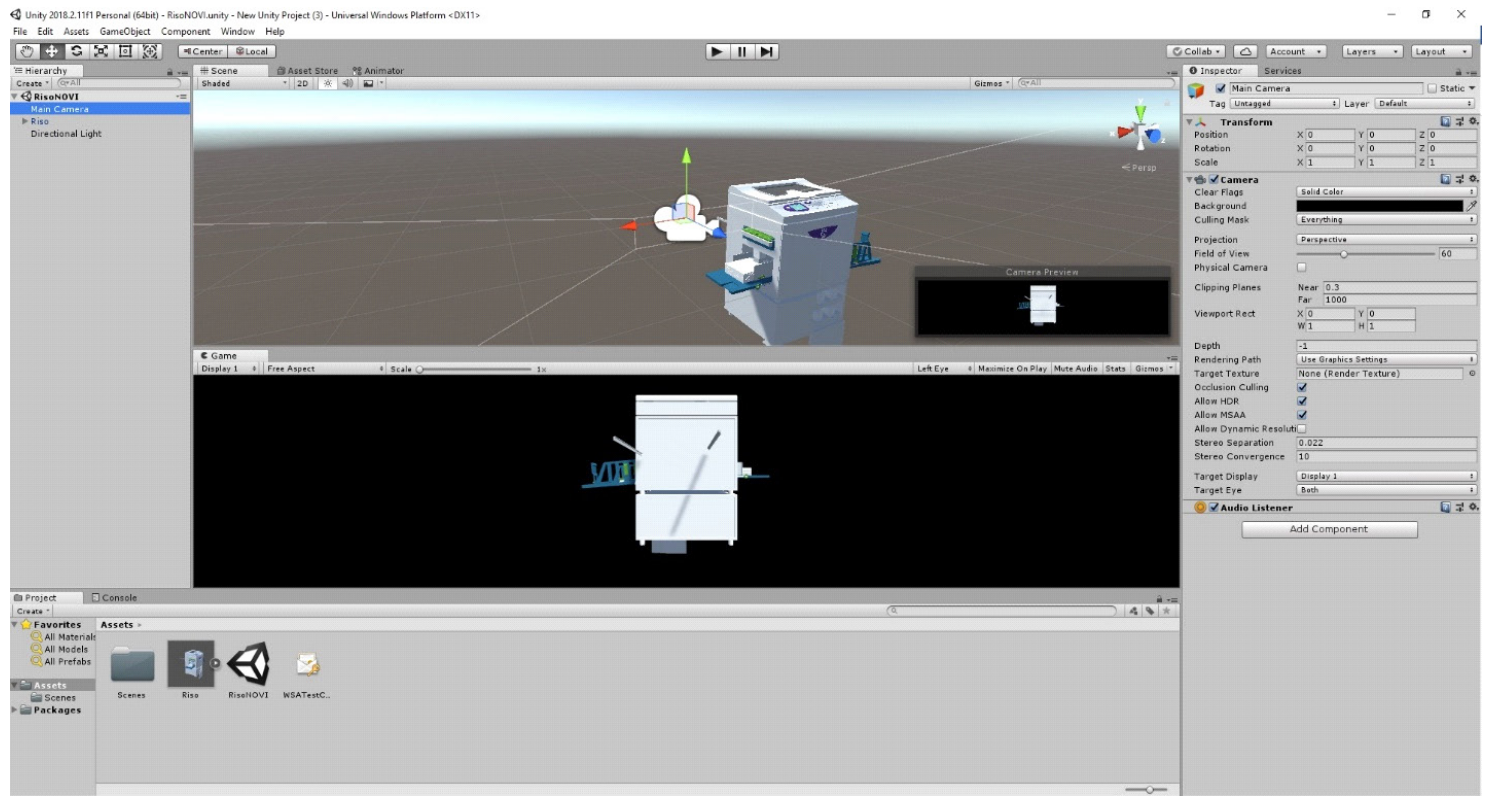

Figure 4: Virtual digital machine transferred to Unity where the camera is added 
The settings that are executed are most related to the camera's position. A pre-defined Main Camera can be used, which is positioned at the $x=0, y=0$ and $z=0$, while the object position is adjusted depending on the desired distance from the viewer. In the object's settings (when the object is selected), the value of the $z$ position determines at what distance the observer will be located. In this case, a value of 2 is set, indicating that the viewer will have the feeling that the object is 2 meters away from it.

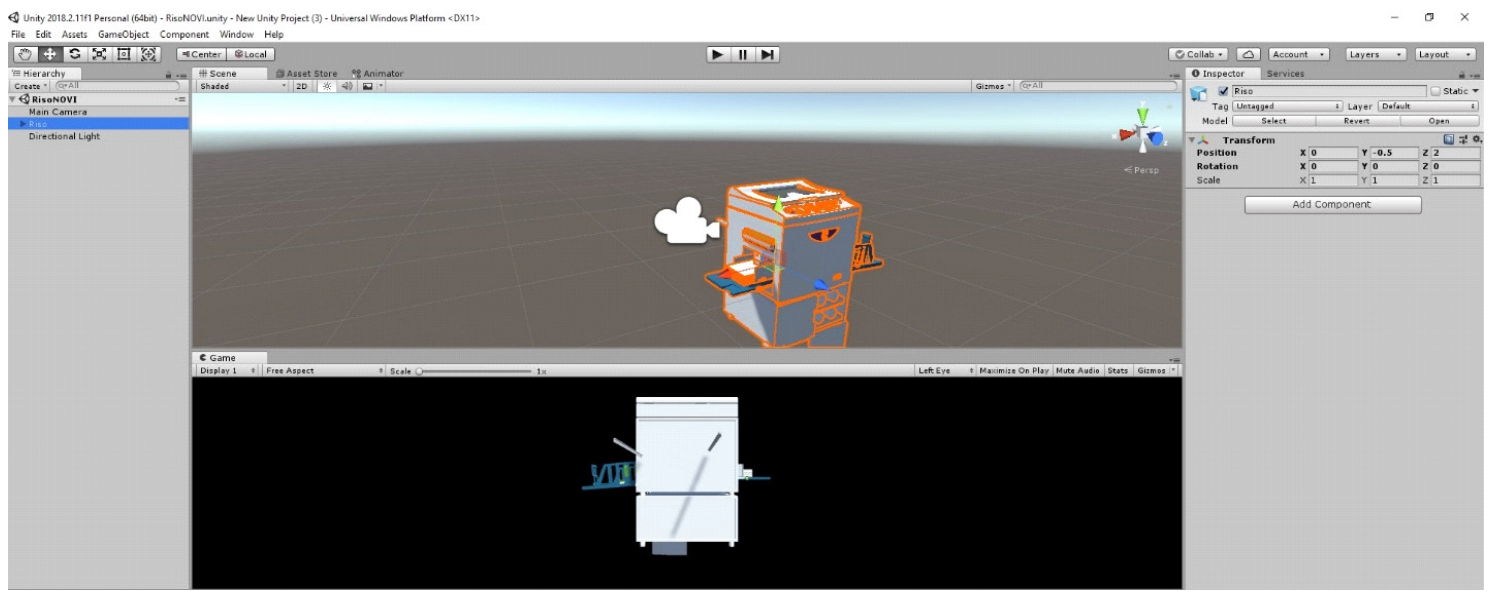

Figure 5: Positioning camera and the model at the right distance is crucial for optimal hologram viewing

After setting the object, it is necessary to create the Build scene and open it in Visual Studio software. It is necessary to select the appropriate options for the Build scene:

- You need to choose the Windows Store platform, SDK Universal 10.

- $\quad$ For Target device it is necessary to choose Any device or HoloLens.

- UWP Build Type should be D3D

- UWP SDK should be selected Latest installed.

- $\quad$ Then, under the Debugging option, it is necessary to select Unity C \# Projects.

After this, Build can be created by clicking on the button with the same name.

After executing settings in the Visual Studio software, it is possible to simulate the model within the emulator or upload the model to the HoloLens device.

\section{CONCLUSION}

Will wearing the entire computer on the head during the work day or after it comes into fashion? Definitely not. Is the connection between the digital and the real world opening up a new door to a better interaction between the computer and the user, and whether the user will have greater benefits in that interaction? Definitely yes. If the benefits of using such devices would be great enough to direct investment in the development of the technology of mixed reality, we can expect more, smaller and smarter devices that will become imperceptible at some point in the future.

Whether the benefits that such devices offer, in the field of education, will be sufficient to trigger their development or whether the game industry will have interest in a mixed reality to support evolutionary progress remains to be seen. Innovative ideas, software, new applications and its development of such devices no longer depend on one team. Research and development modules of the device and their open platform enable everyone to become part of the development team. Such an approach will certainly accelerate the development and application of mixed reality devices.

Researchers in educational institutions can contribute to the development of mixed reality devices by exploring the benefits of mixed reality on education and other real-world problems.

\section{ACKNOWLEDGEMENT}

The research is supported by the Ministry of Education, Science and Technology Development of the Republic of Serbia, project number: 35027 "Development of software model for scientific and production improvement in graphic industry". 


\section{REFERENCES}

[1] Barraclough, A., Guymer, I.: "Virtual reality - A role in environmental engineering education?", Water Science \& Technology, 38 (11), 303-310, 1998. doi: 10.1016/S0273-1223(98)00668-4

[2] Bray, B., Zeller, M.: "What is mixed reality", URL: https://docs.microsoft.com/en-us/windows/mixedreality (last request: 2018-10-1)

[3] Bruner, J. S.: "Toward a Theory of Instruction", (Oxford, Oxford University Press, 1966.)

[4] Mayer, R. E.: "Multimedia learning", 2nd ed, (New York, Cambridge University Press, 2009.)

[5] Oxland, K.: "Gameplay and Design" (Harlow, Addison-Wesley, 2004.)

[6] Pinćjer, I., Tomić, I.: "Interactive Educational Tool for Digital Printing System", Innovations in Publishing, Printing and Multimedia Technologies (Kauno Kolegija, Kaunas, 2018), pages 106-112

[7] Race, P.: "Making Learning Happen", 3rd ed, (London, Sage. 2014.)

[8] Tavanti, M., Lind, M.: "2D vs 3D, implications on spatial memory", Proceedings of IEEE Symposium on Information Visualization, 2001. (INFOVIS 2001., San Diego, 2001), pages 139-145.

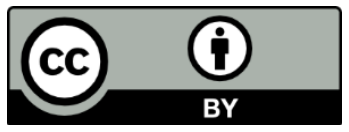

(C) 2018 Authors. Published by the University of Novi Sad, Faculty of Technical Sciences, Department of Graphic Engineering and Design. This article is an open access article distributed under the terms and conditions of the Creative Commons Attribution license 3.0 Serbia (http://creativecommons.org/licenses/by/3.0/rs/). 